8 Larson RA, Druker BJ, Guilhot F, O'Brien SG, Riviere GJ, Krahnke T et al. Imatinib pharmacokinetics and its correlation with response and safety in chronic-phase chronic myeloid leukemia: a subanalysis of the IRIS study. Blood 2008; 111: 4022-4028.

9 Picard S, Titier K, Etienne G, Teilhet E, Ducint D, Bernard MA et al. Trough imatinib plasma levels are associated with both cytogenetic and molecular responses to standard-dose imatinib in chronic myeloid leukemia. Blood 2007; 109: 3496-3499.

10 Shah NP, Kasap C, Weier C, Balbas M, Nicoll JM, Bleickardt E et al. Transient potent $B C R-A B L$ inhibition is sufficient to commit chronic myeloid leukemia cells irreversibly to apoptosis. Cancer Cell 2008; 14: 485-493.

11 Snead JL, O'Hare T, Adrian LT, Eide CA, Lange T, Druker BJ et al. Acute dasatinib exposure commits Bcr-Abl-dependent cells to apoptosis. Blood 2009; 114: 3459-3463.

12 Hiwase DK, White DL, Saunders VA, Kumar S, Melo JV, Hughes TP. Short-term intense Bcr-Abl kinase inhibition with nilotinib is adequate to trigger cell death in BCR-ABL $(+)$ cells. Leukemia 2009; 23: 1205-1206.

13 Lipka DB, Wagner M-C, Dziadosz M, Schnöder T, Heidel F, Schemionek M et al. Intracellular retention of $\mathrm{ABL}$ kinase inhibitors determines commitment to apoptosis in CML cells. PLoS One 2012; 7: e40853.

14 Hegedűs C, Özvegy-Laczka C, Apáti Á, Magócsi M, Német K, Öfi L et al. Interaction of nilotinib, dasatinib and bosutinib with $A B C B 1$ and $A B C G 2$ : implications for altered anti-cancer effects and pharmacological properties. Br J Pharmacol 2009; 158: $1153-1164$

15 Dohse M, Scharenberg C, Shukla S, Robey RW, Volkmann T, Deeken JF et al. Comparison of ATP-binding cassette transporter interactions with the tyrosine kinase inhibitors imatinib, nilotinib, and dasatinib. Drug Metab Disposition 2010; 38: $1371-1380$.

16 Dziadosz M, Wagner M-C, Lipka DB, Fischer T, Bartels H. High-performance liquid chromatography with ultraviolet detection and protein precipitation as a way of quantitative determination of nilotinib with and without internal standard. J Liq Chromatogr Relat Techno 2012.

17 Weisberg E, Manley PW, Breitenstein W, Bruggen J, Cowan-Jacob SW, Ray A et al. Characterization of AMN107, a selective inhibitor of native and mutant Bcr-Abl. Cancer Cell 2005; 7: 129-141.

18 Manley PW, Cowan-Jacob S, Fendrich G, Liebetanz J, Mestan J, Martin N, Fabbro D. 100th Annual Meeting of the American Association for Cancer Research 18-22 April, 2009; Abstract no. 3715.

19 Manley PW, Cowan-Jacob SW, Fendrich G, Jahnke W, Fabbro D. Nilotinib, in comparison to both dasatinib and imatinib, possesses a greatly prolonged residence time when bound to the BCR-ABL kinase SH1 domain. Blood (ASH Annu Meet Abstr) 2011; 118: 1674

\title{
OPEN
}

\section{Initially disadvantaged, TEL-AML1 cells expand and initiate leukemia in response to irradiation and cooperating mutations}

Leukemia (2013) 27, 1570-1573; doi:10.1038/leu.2013.15

Although TEL-AML1 is present in one-fourth of childhood precursor B-cell acute lymphoblastic leukemia (pre-B ALL), the expression of the ETV6-RUNX1 gene encoding this fusion protein has been a poor initiator of leukemia in multiple mouse models. ${ }^{1-8}$ To gain insight into why this common lesion does not readily initiate disease, we took advantage of a novel mouse model, in which TEL-AML1 expression was linked to a fluorescence marker and could be induced in a subset of hematopoietic cells during embryonic development. The laboratory of Corrinne Lobe had generated a reporter mouse in which enhanced green fluorescent protein (EGFP) was expressed following Cre-mediated excision (designated herein as ZEG mice), and subsequently generated an analogous mouse, in which a human TEL-AML1 encoding complementary DNA and an internal ribosomal entry site were placed upstream of EGFP (designated herein as TA1 mice). We crossed the inducible ZEG and TA1 mice with mice expressing Cre under the control of the Tie2(Tek) promoter, which is expressed in endothelial cells as well as during early hematopoietic development. These crosses resulted in mice carrying either the Tie2-Cre and inducible ZEG transgenes (Cre/ZEG mice) or the Tie2-Cre and inducible TA1 transgenes (Cre/TA1 mice). Mice carrying the inducible TA1 transgene but lacking Cre were also generated (Cre-/TA1 + control mice).

The Tie2-Cre cassette resulted in EGFP expression in most, but not all, mature hematopoietic cells of Cre/ZEG mice (Figures 1a and $b$ ). The fact that Tie2-Cre resulted in variegated EGFP expression enabled us to determine whether TEL-AML1 expression had a selective advantage or disadvantage on hematopoietic development. If TEL-AML1 provided a selective advantage in Cre/ TA1 mice, we expected the percentage of EGFP expressing cells to be increased relative to that observed in the Cre/ZEG mice. However, if TEL-AML1 provided a selective disadvantage, EGFP expressing cells would be decreased relative to the Cre/ZEG control. We initially surveyed 5-week-old mice for induction of EGFP in various hematopoietic tissues (Figure 1b). We did not observe any cell type in which TEL-AML1 provided a selective advantage. There was a clear selective disadvantage of TEL-AML1 in B-cells and in T-cells. TEL-AML1 had a modest negative impact on the development of bone marrow myeloid cells and, interestingly, was somewhat tolerated by peritoneal B-cells.

We also took advantage of mice that were part of our survival study (see below) to assess how TEL-AML1 impacted hematopoiesis in aging mice. TEL-AML1 did not impact the fitness of hematopoietic stem and most progenitor cells, but was selected against in megakaryocyte-erythroid progenitors (MEP, Figure 1c). Results in these aged mice confirmed a selective disadvantage in maturing lymphocytes, as well as a sizeable disadvantage during granulopoiesis in older mice (Figure 1d). The substantially impaired contribution to granulopoiesis in older mice might reflect an interaction of TEL-AML1 with the shifting hematopoietic stem/early progenitor numbers and lineage output that occur in aged mice. ${ }^{9,10}$ However, as shown in Supplementary Figure S1, TEL-AML1 expression had no impact on survival.

Low-dose radiation can cause lymphoid tumors in mice (Gross et al. ${ }^{11}$ and references therein). We irradiated Cre/ZEG and Cre/TA 1 mice at 5 and 6 weeks of age with $1.7 \mathrm{~Gy}$. We observed the impact of this irradiation on the percentage of cells in the peripheral blood that expressed EGFP and on survival. Cre/ZEG mice had a high percentage of EGFP expressing cells in their peripheral blood whether or not they were irradiated (Figure 1e). This high percentage of EGFP was seen in cells with low side scatter (primarily lymphocytes) and cells with higher side scatter (primarily granulocytes). In contrast, Cre/TA1 mice had low levels of EGFP in the blood before irradiation (greater expression of EGFP was observed in granulocytes than in lymphocytes), and the percentage of GFP increased greatly in the blood (in both the low and higher side scatter blood cells) when assessed 5-14 months after irradiation. Irradiation curtailed survival and induced tumors in both the Cre/ZEG and Cre/TA1 
a

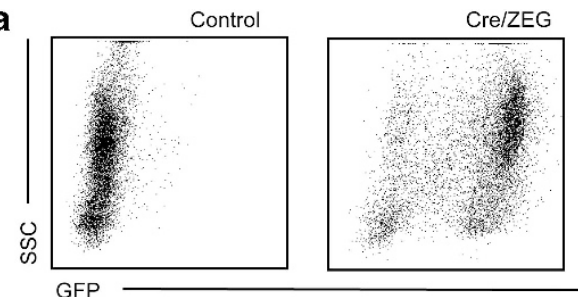

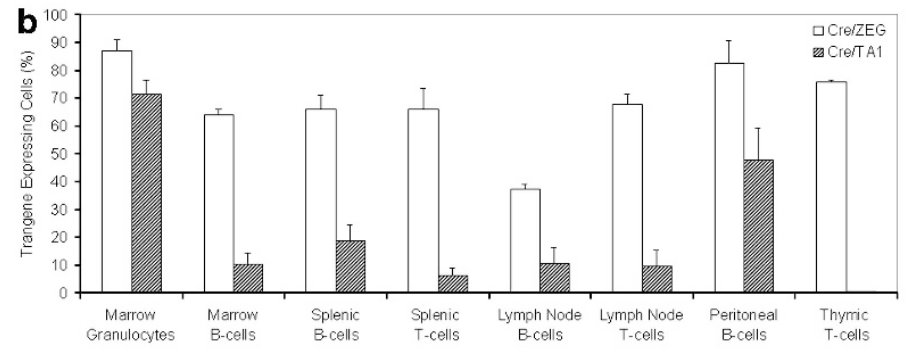
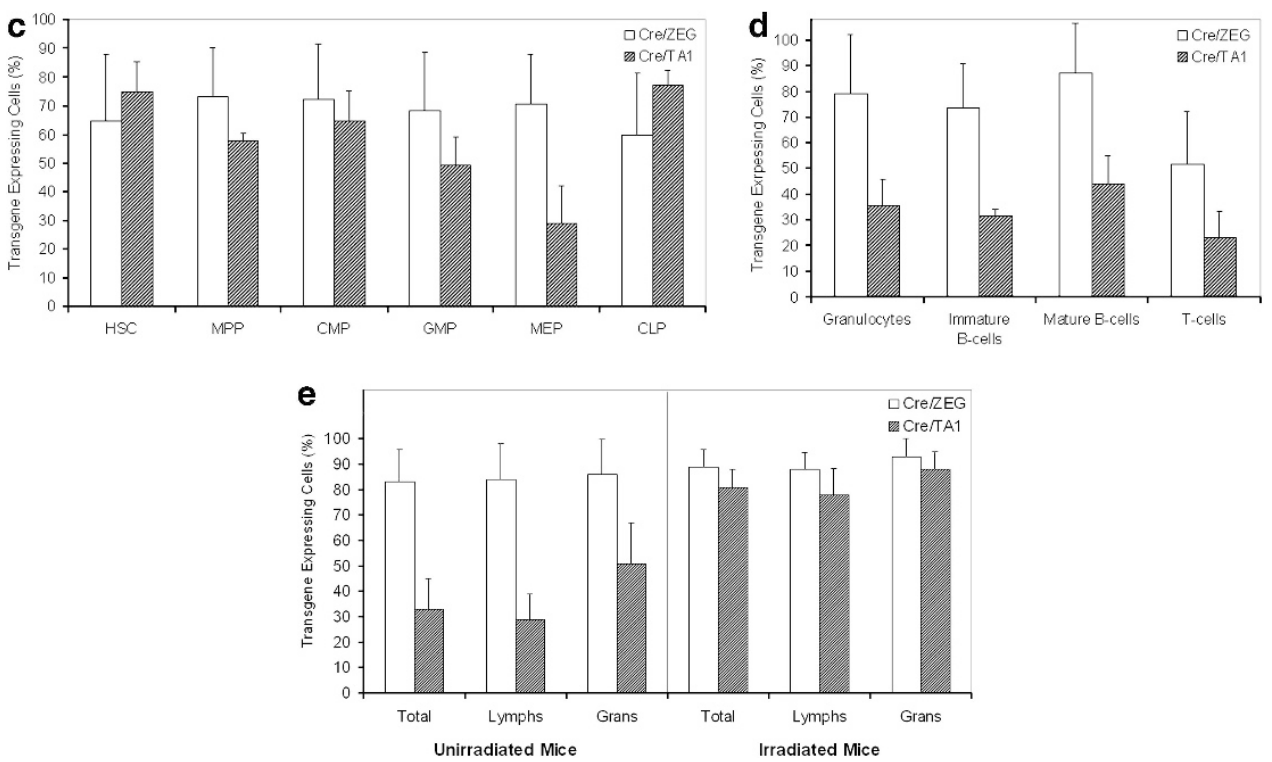

Figure 1. Selective disadvantage to maturing hematopoietic cells that express TEL-AML1 is reversed after irradiation. (a) Representative flow cytometry plots of bone marrow from aged control, Cre/ZEG and Cre/TA1 mice showing variegated transgene expression. (b) Percentage of maturing cell populations that express the EGFP marker (indicating transgene expression) in 5-week-old Cre/ZEG and Cre/TA1 mice, mean \pm s.d. $n=3$ per group. Differences between groups are statistically significant for all populations $(P<.05)$. Granulocytes: Gr $1+/$ Mac- $1+$ cells. B-cells: CD19 + cells. T-cells: CD3 + cells for spleen and lymph node, CD5 + cells for thymus. (c) Percentage of stem and progenitor cell populations that express the EGFP marker (indicating transgene expression) in aged Cre/ZEG and Cre/TA1 mice (19-21 months), mean \pm s.d. $n=3$ per group. The only statistically significant difference between the groups is for MEP $(P<.05)$. HSC: Lin-, C-kit + , Sca- $1+$, Flk2-, CD48-, CD150 + . MPP: Lin-, C-kit + , Sca-1 + , Flk2 + . CMP: Lin-, C-kit + , Sca-1-, CD34 +, FcgR-. GMP: Lin-, C-kit + , Sca-1-, CD34 + , FcgR + . MEP: Lin-, C-kit + , Sca-1-, CD34-, FcgR-. CLP: Lin-, Flk2 +, IL7-R +, C-kit lo, Sca-1 lo. (d) Percentage of maturing cell populations that express the EGFP marker (indicating transgene expression) in aged Cre/ZEG and Cre/TA1 mice (19-21 months), mean \pm s.d. $n=3$ per group. Differences between groups are statistically significant for Gr1 +/Mac- $1+$ granulocytes, B220 +/CD19+/lgM- immature B-cells, and B220 +/CD19+/ $\operatorname{lgM}+$ mature B-cells $(P<.05)$, and trend towards statistical significance for CD3 + T-cells $(P=.06)$. (e) Percentage of blood cells that expressed the EGFP marker (indicating transgene expression) in unirradiated or irradiated Cre/ZEG and Cre/TA1 mice, mean \pm s.d. Percentages are shown for total blood cells, as well as for subpopulations: cells with low side-scatter (predominantly lymphocytes, Lymphs) and cells with higher sidescatter (granulocytes, Grans). Unirradiated mice were 2-16 months of age. Irradiated mice had received 1.7 Gy of gamma irradiation at 5 weeks and again at 6 weeks of age and were studied when 5-14 months of age. Data were derived from 10 unirradiated and 15 irradiated Cre/ZEG mice and from 14 unirradiated and 11 irradiated Cre/TA1 mice. The differences seen between Cre/ZEG and Cre/TA1 are statistically significant for all comparisons ( $P \leqslant .00001$ for all comparisons excepting 'postirradiation, all cells' for which $P=.009)$. There were no statistically significant differences when Cre/ZEG unirradiated mice were compared with Cre/ZEG irradiated mice. In contrast, irradiation led to an increase in EGFP expressing cells in the total, low SSC and high SSC cell populations $(P<.00001)$.

mice (Supplementary Figure S2), but no B-cell leukemias or lymphomas were observed.

CDKN2A is lost in approximately one-third of ALL overall and in one-fourth of leukemias expressing TEL-AML1. ${ }^{12}$ We therefore sought to assess possible cooperation between TEL-AML1 and
Cdkn2a loss in mice. The initial description of Cdkn2a null mice noted the presence of lymphomas, including B-cell lymphomas in these mice ${ }^{13}$ but the characterization of this disease was limited. We therefore assessed the incidence and some of the features of these lymphomas in Cdkn2a null mice. As shown in the right hand 

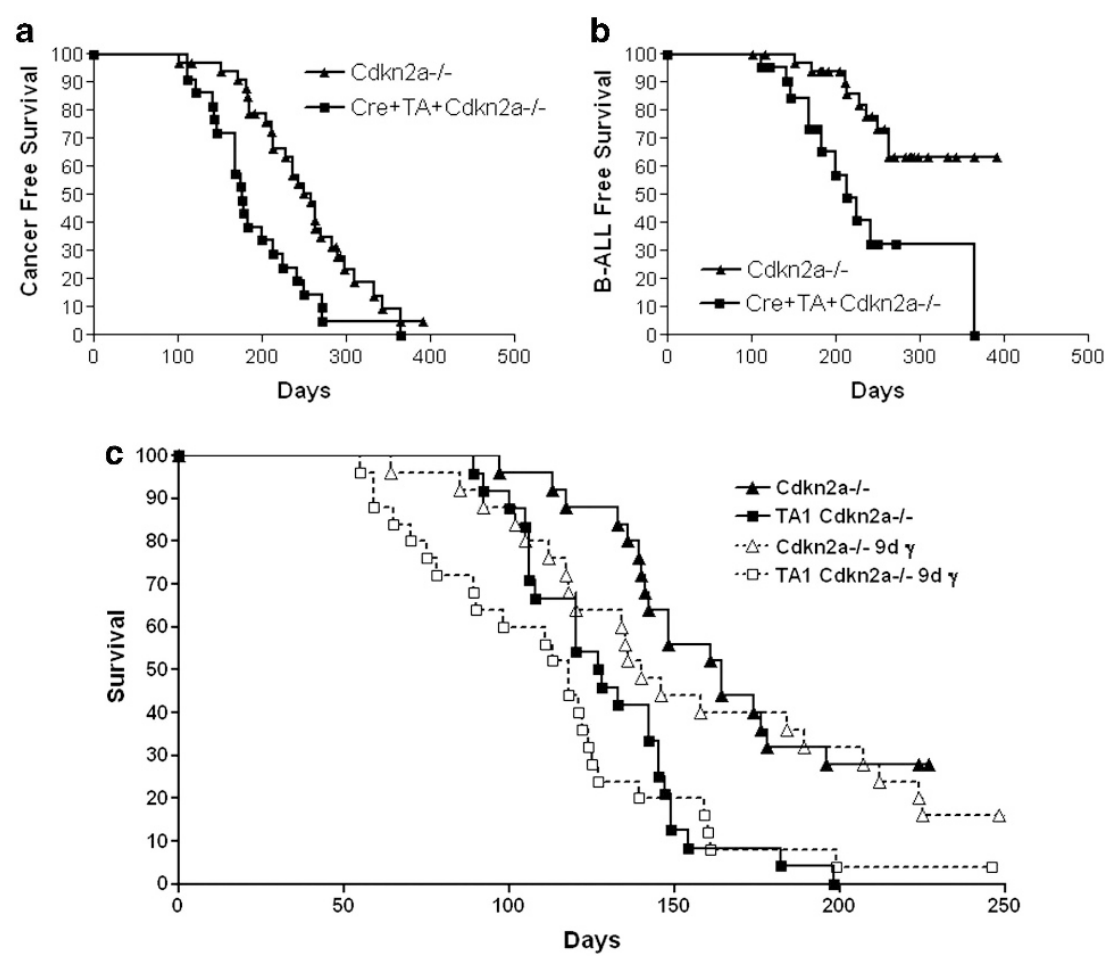

Figure 2. Cdkn2a null mice that express TEL-AML1 develop a high incidence of B-cell lymphoblastic lymphoma/leukemia which is accelerated by irradiation. (a) Cohorts of Cdkn2a null mice $(n=34)$ and Cre/TA1 Cdkn2a null mice $(n=22)$ were followed for the development of malignancies. Curves show decreased cancer-free survival of mice that express TEL-AML1 (median cancer free survival for Cre/TA1 Cdkn2a null vs $C d k n 2 a$ null 177 vs 259 days, $P=.002$ ). One mouse in the Cre/TA1 Cdkn2a null cohort and four mice in the Cdkn2a null cohort did not have evidence of malignancy at pathologic examination and were censored at the time of death. Results were similar if total survival of these mice was assessed (see Material and Methods). (b) Survival curves showing the development of B-cell neoplasms in the cohorts described for panel A. $P=.003$ for decreased survival due to B-ALL (including precursor B-cell lymphoblastic lymphoma/leukemia and Burkitt like lymphoma). (c) Cdkn2a null mice and Cre/TA1 Cdkn2a null mice were irradiated with $1.88 \mathrm{~Gy}$ of gamma radiation at 9 days of age or were not irradiated. At 5 weeks of age their bone marrows were collected and transplanted into lethally irradiated congenic animals. Recipients of marrow from unirradiated mice and from irradiated mice were followed for the development of disease ( $n=24$ for Cre/TA1 Cdkn $2 a$ null unirradiated mice, $n=25$ for three other groups). Irradiation was associated with early disease development in Cre/TA1 Cdkn2a null animals (see text).

curves in Figure 2, Cdkn2a null mice died of malignancies with a median latency of 259 days (Figure 2a) and B-cell lymphoma/ leukemia was observed in 9 of 34 mice followed as a cohort (Figure 2b). We performed flow cytometric immunophenotyping (Supplementary Table) of eight of these samples as well as an additional seven B-cell lymphomas that arose in Cdkn2a null mice and that we had collected from selected non-cohort animals (we had previously sporadically assessed ill mice in our Cdkn2a colony and had identified lymphomas in a number of these animals). These analyses showed the samples to be uniformly $\mathrm{CD} 45 \mathrm{R}(\mathrm{B} 220)+, \mathrm{CD} 19+, \mathrm{CD} 43+$, with 11 of 15 samples lacking surface immunoglobulin (Ig) $M$ (and hence compatible with pre-B LBL, precursor B-cell lymphoblastic lymphoma/leukemia) ${ }^{14}$ and four of 15 samples showing some degree of surface IgM expression (and hence compatible with BLL, Burkitt like lymphoma including mature B-cell lymphomas with lymphoblastic morphology). ${ }^{14}$

To assess the interaction of TEL-AML1 with Cdkn2a loss, the Tie2-Cre and inducible TEL-AML1 transgenes were crossed onto the $C d k n 2 a$ null background. As shown in the left hand curves of Figure 2, TEL-AML1 decreased the median cancer free survival of Cdkn2a null mice to 177 days (Figure 2a, $P=.002$ for decreased cancer free survival), and increased the incidence of B-cell lymphoma from $26-50 \%$ (Figure $2 b, 11$ of 22 mice; $P=.003$ for decreased survival due to B-cell disease). Morphologic examination of the lymphomas showed a lymphoblastic morphology and demonstrated infiltrates in the liver and spleen (Supplementary Figure S3). Flow cytometric immunophenotyping of these leukemias demonstrated disease similar to that observed with Cdkn2a alone, including 7 of 11 samples compatible with pre-B LBL and 4 of 11 compatible with BLL (Supplementary Figure S4A and Supplementary Table). All 11 samples expressed weak to moderate levels of EGFP, whereas a mast cell neoplasm and a histiocytic sarcoma that arose in this cohort were more strongly EGFP positive. As expected, TEL-AML1 transcripts could be detected in B-cell lymphoma from TEL-AML1 Cdkn2a null mice but not in lymphoma that arose in Cdkn2a null mice in the absence of TEL-AML1 (Supplementary Figure S4B). In addition, we were able to demonstrate expression of TEL-AML1 protein by western blot in a lymphoma with moderate EGFP expression (Supplementary Figure S4C).

We assessed the potential for radiation exposure to enhance leukemia development in these mice. We exposed 9-day-old pups to $1.88 \mathrm{~Gy}$ or did not irradiate them. At 5 weeks of age, bone marrows were harvested and analyzed by flow cytometry before transplantation into lethally irradiated congenic recipient animals. We observed that irradiation fostered outgrowth of TEL-AML1 expressing cells in Cdkn2a null mice: without irradiation, $35 \%$ of bone marrow CD45R + /CD19 + /lgM- immature B-cells expressed EGFP, whereas in irradiated mice $76 \%$ of these immature B-cells expressed EGFP ( $n=5$ per group, $P=.0002$ ). Survival curves (Figure 2c) provided further confirmation of the leukemogenic effect of TEL-AML1 in Cdkn2a null mice: median survival for recipients of unirradiated Cre/TA1 Cdkn2a null marrow was 128 days vs 164 days for $C d k n 2 a$ null unirradiated marrow $(P=.0003)$; median survival for recipients of irradiated Cre/TA1 Cdkn2a null 
marrow was 118 days vs 140 days for Cdkn2a null irradiated $(P=.009)$. In all four groups, the majority of deaths were due to B-cell lymphoma/leukemia.

We had hypothesized that irradiation would accelerate the development of disease in at least some of the recipient animals. Although there was substantial overlap of the survival curves of genetically matched irradiated and unirradiated groups, the survival curves of the irradiated groups appeared shifted to the left as compared with the respective unirradiated groups. Using the shortest time to leukemia in the control Cdkn2a null unirradiated group for comparison (97 days) we observed that 9 of 25 mice in the Cre/TA1 Cdkn2a irradiated group developed disease prior to this time, whereas only 2 of 24 mice in the Cre/TA1 Cdkn2a unirradiated group showed early disease $(P=.02$; see Materials and Methods for statistical discussion). This finding suggests that some mice received cells for which low-dose radiation caused mutations that cooperated with TEL-AML1 expression and Cdkn2a loss to induce leukemia. Our results provide support for a model of TEL-AML1 leukemogenesis, in which environmental exposures may both enable expansion of initially disadvantaged TEL-AML1 expressing cells and cause cooperative leukemogenic mutations.

\section{CONFLICT OF INTEREST}

The authors declare no conflict of interest.

\section{ACKNOWLEDGEMENTS}

This work was funded by the Electric Power Research Institute.

$$
\begin{aligned}
& \mathrm{M} \mathrm{Li}^{1} \text {, L Jones }{ }^{1}, \mathrm{C} \text { Gaillard }{ }^{1}, \mathrm{M} \text { Binnewies }{ }^{2}, \mathrm{R} \text { Ochoa }^{1,6}, \mathrm{E} \mathrm{Garcia}^{1} \text {, } \\
& \text { V Lam }{ }^{3}, \mathrm{G} \mathrm{Wei}^{4,1}, \mathrm{~W} \text { Yang }^{5} \text {, C Lobe }{ }^{5} \text {, M Hermiston }{ }^{3} \text {, E Passegué }{ }^{2} \\
& \text { and SC Kogan }{ }^{1}
\end{aligned}
$$

${ }^{6}$ Present address: Department of Pediatrics, Stanford University School of Medicine, Stanford, CA, USA.

\section{REFERENCES}

1 Andreasson P, Schwaller J, Anastasiadou E, Aster J, Gilliland DG. The expression of ETV6/CBFA2 (TEL/AML1) is not sufficient for the transformation of hematopoietic cell lines in vitro or the induction of hematologic disease in vivo. Cancer Genet Cytogenet 2001; 130: 93-104.

2 Bernardin F, Yang Y, Cleaves R, Zahurak M, Cheng L, Civin Cl et al. TEL-AML1, expressed from $t(12 ; 21)$ in human acute lymphocytic leukemia, induces acute leukemia in mice. Cancer Res 2002; 62: 3904-3908.

3 Morrow M, Horton S, Kioussis D, Brady HJ, Williams O. TEL-AML1 promotes development of specific hematopoietic lineages consistent with preleukemic activity. Blood 2004; 103: 3890-3896.

4 Tsuzuki S, Seto M, Greaves M, Enver T. Modeling first-hit functions of the $t(12 ; 21)$ TEL-AML1 translocation in mice. Proc Natl Acad Sci USA 2004; 101: 8443-8448.

5 Fischer M, Schwieger M, Horn S, Niebuhr B, Ford A, Roscher S et al. Defining the oncogenic function of the TEL/AML1 (ETV6/RUNX1) fusion protein in a mouse model. Oncogene 2005; 24: 7579-7591.

6 Hong D, Gupta R, Ancliff P, Atzberger A, Brown J, Soneji S et al. Initiating and cancer-propagating cells in TEL-AML1-associated childhood leukemia. Science 2008; 319: 336-339.

7 Schindler JW, Van Buren D, Foudi A, Krejci O, Qin J, Orkin SH et al. TEL-AML1 corrupts hematopoietic stem cells to persist in the bone marrow and initiate leukemia. Cell Stem Cell 2009; 5: 43-53.

8 van der Weyden L, Giotopoulos G, Rust AG, Matheson LS, van Delft FW, Kong J et al. Modeling the evolution of ETV6-RUNX1-induced B-cell precursor acute lymphoblastic leukemia in mice. Blood 2011; 118: 1041-1051.

9 Rossi DJ, Bryder D, Zahn JM, Ahlenius H, Sonu R, Wagers AJ et al. Cell intrinsic alterations underlie hematopoietic stem cell aging. Proc Natl Acad Sci USA 2005; 102: 9194-9199.

10 Pang WW, Price EA, Sahoo D, Beerman I, Maloney WJ, Rossi DJ et al. Human bone marrow hematopoietic stem cells are increased in frequency and myeloid-biased with age. Proc Natl Acad Sci USA 2011; 108: 20012-20017.

11 Gross L, Dreyfuss Y. Inhibition of the development of radiation-induced leukemia in mice by reduction of food intake. Proc Natl Acad Sci USA 1986; 83: 7928-7931.

12 Mullighan CG, Goorha S, Radtke I, Miller CB, Coustan-Smith E, Dalton JD et al. Genome-wide analysis of genetic alterations in acute lymphoblastic leukaemia. Nature 2007; 446: 758-764.

13 Serrano M, Lee H, Chin L, Cordon-Cardo C, Beach D, DePinho RA. Role of the INK4a locus in tumor suppression and cell mortality. Cell 1996; 85: 27-37.

14 Morse HC 3rd, Anver MR, Fredrickson TN, Haines DC, Harris AW, Harris NL et al. Bethesda proposals for classification of lymphoid neoplasms in mice. Blood 2002; 100: $246-258$.

(c) (i) $\odot$ This work is licensed under a Creative Commons Attributioncc. NonCommercial-NoDerivs 3.0 Unported License. To view a copy of this license, visit http://creativecommons.org/licenses/by-nc-nd/3.0/

\section{Landmark analysis of DNMT3A mutations in hematological malignancies}

Leukemia (2013) 27, 1573-1578; doi:10.1038/leu.2013.65

Methylation of CpG islands is an epigenetic modification that is important for cellular development. ${ }^{1}$ Different members of the DNMT gene family are involved in de novo CpG methylation. The DNMT3A gene is located on chromosome 2p23.3 and codes for a DNA methyltransferase. Ley et al. ${ }^{1}$ and Yan et al. ${ }^{2}$ were able to unravel DNMT3A mutations (DNMT3Amut) in acute myeloid leukemia (AML), thus supporting a role for aberrant DNA methylation to contribute to the pathogenesis of this disease. Subsequent studies detected DNMT3Amut in 17-34\% of AML with normal karyotype (CN-AML) (see Table 1). Depending on the respective patient cohort characteristics, occurrence of the mutations was associated with an inferior survival (Table 1). Subsequently, DNMT3Amut were studied and observed in other hematological malignancies (Table 1): mutation frequencies were 